\title{
Predator-Prey-Subsidy Population Dynamics on Stepping-Stone Domains
}

\author{
Lulan Shen and Robert A. Van Gorder* \\ Mathematical Institute, University of Oxford, Andrew Wiles Building, Radcliffe Observatory Quarter, Woodstock Road, Oxford, OX2 6GG, UK \\ *Robert.VanGorder@maths.ox.ac.uk
}

\begin{abstract}
Predator-prey-subsidy dynamics on stepping-stone domains are examined using a variety of network configurations. Our problem is motivated by the interactions between arctic foxes (predator) and lemmings (prey) in the presence of seal carrion (subsidy) provided by polar bears. We use the $n$-Patch Model, which considers space explicitly as a "Stepping Stone" system. We consider the role that the carrying capacity, predator migration rate, input subsidy rate, predator mortality rate, and proportion of predators surviving migration play in the predator-prey-subsidy population dynamics. We find that for certain types of networks, added mobility will help predator populations, allowing them to survive or coexist when they would otherwise go extinct if confined to one location, while in other situations (such as when sparsely distributed nodes in the network have few resources available) the added mobility will hurt the predator population. We also find that a combination of favorable conditions for the prey and subsidy can lead to the formation of limit cycles (boom and bust dynamic) from stable equilibrium states. These modifications to the dynamics vary depending on the specific network structure employed, highlighting the fact that network structure can strongly influence the predator-prey-subsidy dynamics in stepping-stone domains.
\end{abstract}

Keywords: predator-prey dynamics; allochthonous resource subsidy; population dynamics; non-equilibrium dynamics; network structure in ecology; stepping-stone model

\section{Introduction}

The Arctic is home to a unique ecosystem with many terrestrial and marine animals, and plant-life. One species living in the Arctic is the arctic fox (Alopex lagopus), a predator. The arctic fox's diet consists of lemmings (Cricetidae family), and certain birds and bird eggs [26, 27]. They also consume seal (Phocidae family) carrion, a nutritional subsidy discarded by polar bears (Ursus martimus) [26, 23]. The arctic fox is a migratory animal, travelling from areas where lemmings breed to areas in which polar bears live. The arctic fox migrates every 3-4 years in September following random paths instead of a specific pattern [36] and migration usually finishes by midFebruary. During its migration, there is a high death rate due to lack of food, trapping, and diseases such as rabies [36], yet during the winter, when migration does not occur, these are still factors in the death rate [24].

Single-species models relevant to laboratory studies in particular can reflect telescoping effects influencing population dynamics in the real world [22]. Verhulst proposed that a selflimiting process should operate when a population becomes too large [22, 33], and suggested logistic growth in a population. The resulting Logistic Equation was later derived from first principles by Lotka [3, 17], and was first used in a model to describe two species competing for the same resources [2]. Shortly after this, Lotka and Volterra derived the first model of predator-prey interactions, known as the Lotka-Volterra Model $[22,34]$, under the assumptions: (i) the prey without predation grows unboundedly in a Malthusian way; (ii) predation reduces the prey's per capita growth rate by a term proportional to the prey and predator populations; (iii) in the absence of any prey for sustenance the predator's death rate can be modeled as an exponential decay function; (iv) the prey's contribution to the predators' growth rate is proportional to the available prey as well as the population of the predator. This model unrealistically predicts that the prey will grow without bound in the absence of predators. For this reason, the inclusion of the logistic equation to prevent the prey from growing in an unbounded manner is often used in variants of the Lotka-Volterra equations.

One variation to the Lotka-Volterra model was suggested by Solomon [31], and a decade later by Holling [9, 10]. Since there is a limit to the amount of prey which can be consumed by a predator in a finite amount of time, they proposed that the predator equation should involve growth of a rational functional form [23, 3] involving Holling's Disk Equation [3, 1]. Such a system was the motivation for a predator-prey-subsidy model [23]

$$
\begin{aligned}
& \frac{d x}{d t}=r x\left(1-\frac{x}{k}\right)-\theta\left(\frac{x}{x+s+h}\right) y, \\
& \frac{d s}{d t}=i-\gamma s-\psi\left(\frac{s}{x+s+h}\right) y, \\
& \frac{d y}{d t}=\left(\frac{\epsilon \theta x+\eta \psi s}{x+s+h}\right) y-\delta y,
\end{aligned}
$$

where $x(t), y(t), s(t)$ are the size of the prey, predator, and subsidy populations at each value of time. For a list of the parameters used, see Table 1 . In (1.2), $i$ is the rate at which the 
Table 1: List of model parameters [23, 15]. Values with superscript $(j)$ denote quantities in patch $j$ of the stepping-stone model.

\begin{tabular}{|c|c|}
\hline Parameter & Description (Value) \\
\hline \hline$t$ & Time $([0,10000])$ \\
\hline$k^{(j)}$ & Prey Carrying Capacity $(0.1,0.4,2.4$ or 5$)$ \\
\hline$r^{(j)}$ & Prey Growth Rate $(0.1$ or 0.2$)$ \\
\hline$i^{(j)}$ & Subsidy Input Rate $(0.1)$ \\
\hline$\gamma^{(j)}$ & Subsidy Decay Rate In Patch $j(1)$ \\
\hline$\delta^{(j)}$ & Predator Mortality Rate $(0.1)$ \\
\hline$\alpha^{(j, l)}$ & Migration Rate $j \rightarrow l([0,1])$ \\
\hline$\lambda^{(j, l)}$ & Migration Survival Rate $j \rightarrow l([0,1])$ \\
\hline$h$ & Half Saturation Parameter $(1)$ \\
\hline$\theta$ & Predator-Prey Consumption Rate $(5)$ \\
\hline$\psi$ & Predator-Subsidy Consumption rate $(5)$ \\
\hline$\epsilon$ & Prey Nutritional Value $(0.1)$ \\
\hline$\eta$ & Subsidy Nutritional Value $(0.1)$ \\
\hline
\end{tabular}

subsidy is introduced into the system and $\gamma$ is the rate where the subsidy decays. The final term in (1.2) represents the rate of the subsidy being consumed by the predator, where $\psi$ is the maximum rate at which the predator consumes the subsidy. The first term in (1.3) describes the predator benefiting from its food sources, where $\epsilon$ and $\eta$ are conversion factors. In [23], Nevai and Van Gorder discovered that the autonomous system (1.1)(1.3) permitted coexistence and extinction equilibria, as well as limit cycles for appropriate parameters. In the autonomous model (1.1)-(1.3), [23] found no evidence of chaotic dynamics. This was later extended to include non-autonomous coefficients in order to model seasonality [15], with a variety of non-equilibrium dynamics observed including stable limit cycles, quasi-periodic oscillations, and chaotic oscillations.

Models that investigate the animal populations' migration effects fall into one of three categories: "Island" models [11, 13], "Stepping-Stone" models [13, 14], and continuum models $[11,13,14]$. The "Island" models include a set of patches in which different populations live. All patches are accessible, and migration between patches occurs instantaneously [13]. Therefore, spatial dimensions are not included in the model. This is the most analytically accessible of the three classes [13], used in the two-patch predator-prey-subsidy model in [23], and in models investigating the effect of having refuge for the prey [30]. "Island" models typically use ordinary differential equations (ODEs) and consider linear inter-patch diffusion [23, 13, 14]. The "Stepping-Stone" classification of models is much like the "Island" class. However, the patches are assigned spatial coordinates and spatial dimensions are now explicitly included in the model [13]. Populations can move between each region via discrete diffusion. "Stepping-Stone" models are used in [16] where the authors use game theory to examine how the predator and prey should behave in their respective locations. The important difference between these two models is that the "Stepping-Stone" model will allow us to understand the role spatial structure will determine predator-prey dynamics. Meanwhile, continuum models can also be employed, however they

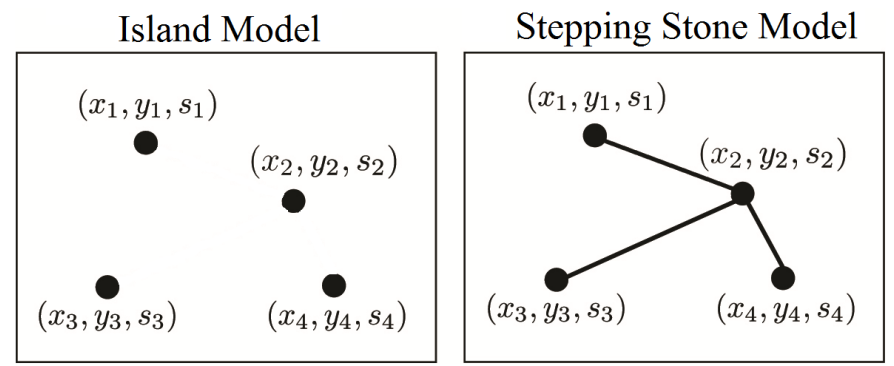

Figure 1: Examples of an 4-patch island model and an 4-patch "Stepping Stone" model.

involve partial differential equations, as they model motion over continuous space, rather than movement between a collection of discrete patches.

In this paper, we shall concern ourselves with Stepping-Stone models. In particular, we are interested in the extension of (1.1)-(1.3) to networks of inter-connected patches. Therefore, we will consider an $n$-Patch Model. A patch is a bounded region of space large enough so each individual prey does not leave its patch, assuming the patches are disconnected. The populations of the prey and subsidy in each patch are not directly influenced by the population in the other patches. The $n$-Patch Model considers space by modelling $n$ patches of the predator, prey and subsidy, where only the predator can migrate between territories. A graph comparing an island model with an $n$-Patch model is shown in Figure 1.

Levy, Harrington and Van Gorder introduced the $n$-Patch model in [15]. In this model, there are $n$ patches, $\left\{\mathbb{P}^{(1)}, \mathbb{P}^{(2)}, \cdots, \mathbb{P}^{(n)}\right\}$. Let $x^{(j)}(t), s^{(j)}(t)$, and $y^{(j)}(t)$ represent the population sizes of the prey, subsidy and predator, respectively, in the patch $\mathbb{P}^{(j)}$ for $j=1, \cdots, n$. When $n=2$, this simplifies to the two-patch model in [23]. It is a $3 n^{\text {th }}$ order system given by the non-dimensionalized system of equations

$$
\begin{aligned}
\frac{d x^{(j)}}{d t}= & r^{(j)} x^{(j)}\left(1-\frac{x^{(j)}}{k^{(j)}}\right)-\theta\left(\frac{x^{(j)}}{x^{(j)}+s^{(j)}+h}\right) y^{(j)}, \\
\frac{d s^{(j)}}{d t}= & i^{(j)}-\gamma^{(j)} s^{(j)}-\psi\left(\frac{s^{(j)}}{x^{(j)}+s^{(j)}+h}\right) y^{(j)}, \\
\frac{d y^{(j)}}{d t}= & \left(\frac{\epsilon \theta x^{(j)}+\eta \psi s^{(j)}}{x^{(j)}+s^{(j)}+h}\right) y^{(j)}-\delta^{(j)} y^{(j)} \\
& +\sum_{l=1}^{n}\left(\lambda^{(l, j)} \alpha^{(l, j)} y^{(l)}-\alpha^{(j, l)} y^{(j)}\right),
\end{aligned}
$$

where, in the $j^{\text {th }}$ patch, $r^{(j)}$ is the intrinsic prey growth rate, $k^{(j)}$ is the carrying capacity of the prey, $i^{(j)}$ is subsidy creation rate, $\gamma^{(j)}$ is the subsidy decay rate, and $\delta^{(j)}$ is the predator mortality rate. The rate where the predator travels from $\mathbb{P}^{(j)}$ to $\mathbb{P}^{(l)}$ is shown by $\alpha^{(j, l)}$. We consider predator migration from a patch to itself unrealistic behavior and set $\alpha^{(j, j)} \equiv 0$ for all $j$. Finally, $\lambda^{(l, j)}$ represents the proportion of predators which survive interpatch migration from $\mathbb{P}^{(l)}$ to $\mathbb{P}^{(j)}$. This is equivalent to unity in [23], which does not consider migration mortality rates. The parameters $\theta, \psi, \epsilon$, and $\eta$ describe the interactions between the predator, the prey, and the subsidy, which we shall assume re- 
main fixed across all patches (for sake of simplicity, as we shall primarily be interested in varying other quantities). The list of state variables and list of parameters are summarized in Tables 2.1 and 2.2 .

The paper [15] only considered simulations for simple $n=2$ or $n=3$ node networks, since the focus of that paper was on seasonality. Still, [15] proved that the dynamics of the general $n$-node system is bounded, although the behavior of the system was not studied. The effects of the subsidy input rate on a two-patch model where the first patch is prey-free and the second patch is subsidy-free were also examined. When the unperturbed system has a limit cycle, increasing the subsidy input rate stabilises the dynamics, and also increases sensitivity of the predator to the subsidy input rate when the prey is extinct. They looked at the average predator mortality rate effects during migration on the three-patch system dynamics where $\mathbb{P}^{(1)}$ is subsidy-free, $\mathbb{P}^{(2)}$ is prey-free and subsidy-free, and $\mathbb{P}^{(3)}$ is preyfree. They found that increasing the average predator mortality rate stabilises the dynamics, and ultimately enables predatormortality and prey-survival [15]. Their results did not consider the influence network structure has over the dynamics of (1.4)(1.6), and this shall be the focus of the present paper.

We shall note that a number of different Stepping-Stone configurations can either push the populations into extinction, or, in some cases, can enrich the dynamics. Rosenzweig theoretically predicted that increasing the supply of limited energy or nutrients tends to be destabilizing [25]. Experiments done by Huffaker et al. [12], Luckinbill [18], Veilleux [32] and Fussmann et al. [4] have demonstated the destablilization of ecosystems following enrichment. Huffaker et al. illustrated that an increase in the food supply to herbivorous mites destabilized their relationship with predatory mites, leading to the extinction of both species $[28,12]$. Thus, attempting to enrich the ecosystem to increase food yield must be approached with caution. There is a risk that this activity may result in decimation of the species [25]. As we shall show, one can destabilize positive steady states in the predator-prey-subsidy model through the modification of the network structure underlying the Stepping-Stone domain.

Our objective is to examine the effect of network structures and discrete diffusion rates on predator-prey-subsidy dynamics of Stepping-Stone models of the form (1.4)-(1.6). The remainder of the paper is organized as follows. In Section 2 we outline the methods used, including the numerical technique, parameter values and initial conditions, and network structures employed. A variety of initial conditions will be used to specify where prey and/or subsidies are located, while the predator is free to move between patches. In Section 3 we give our numerical results, constructing a number of useful bifurcation diagrams for various network configurations and under various configurations of prey and subsidy locations. We summarize these results and discuss their biological implications in Section 4.

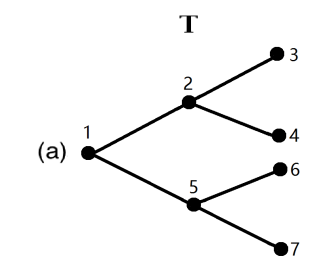

(b)

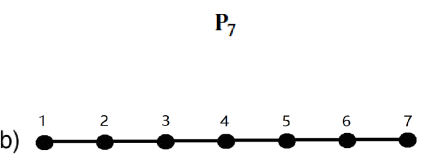

(c)

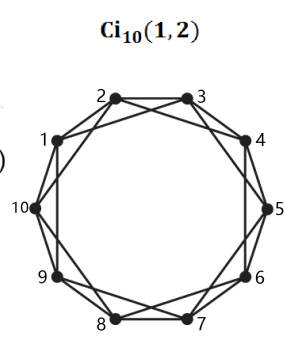

(d)

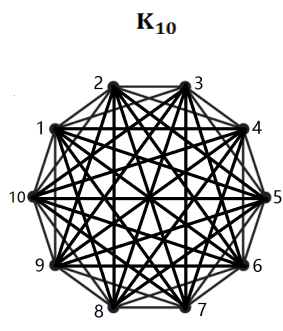

H

(e)

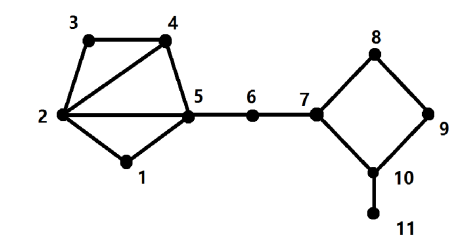

Figure 2: Types of networks used, including (a) a perfect binary tree with seven nodes $(T)$ [29], (b) a path graph with seven nodes $\left(P_{7}\right)$ [29], (c) a 5-antiprism graph with ten nodes $\left(C i_{10}(1,2)\right)$ [8], (d) a complete graph with ten nodes $\left(K_{10}\right)$, and (e) a hybrid network with eleven nodes $(H)$ [21].

\section{Methods}

\subsection{Network structures considered}

To model predator-prey interactions spatially, we have very few empirical examples of precise spatial domains. In particular, there do not exist many studies modelling network structure effects on predator-prey dynamics. The other studies to model biological structures involve models where dispersion was strictly controlled and different habitats were connected through small corridors. In [29], one experiment uses multiple protists and one rotifer species using a perfect binary tree with seven nodes $(T)$ and continuous path graphs with seven nodes $\left(P_{7}\right)$, shown in (a) and (b) in Figure 2. These different networks are used to simulate diversity patterns and dynamics among different colonies to influence the speed and pattern of colonization among $T$ [29].

Network connectivity determines how species can migrate spatially from one habitat to another within a network. With the different bacteria species mentioned in [29], we have a different type of diversity compared to the colonisation of different versus single species of bacteria. However, the diversity of both types of bacteria is higher in $T$ than with $P_{7}$ [29]. Therefore, the network structure will have some influence on population dynamics.

Holland and Hastings discuss dispersal network structures in [8]. They use regular four degree lattices (each node represents a habitat patch connecting to its four closest neighbours) to model dispersal networks. This network is isomorphic to 
the 5-antiprism graph with ten nodes, known as $C i_{10}(1,2)$ and shown in Figure 2(c). The authors mentioned that ten nodes strikes a perfect balance between too few nodes and too complex a lattice. Too few nodes may lead to spurious results, while a complex systems with hundreds of nodes behaves similarly to a system with ten nodes [8]. A four degree lattice is also complex enough to model networking effects with non-neighboring nodes without too much redundancy. The above example can be extended to a complete graph network with ten nodes $\left(K_{10}\right)$, shown in Figure 2(d), where each node for $K_{10}$ is connected with every other node.

Graph theory has been used to model persistent patches that are vital to habitat connectivity and where animal species need conservation the most [21]. In [21], Minor and Urban use graph theory to model the Wood Thrush species' effects on their habitat and environmental changes. We simplified the network of basic graph terminology in [21] by removing two isolated nodes. Then we obtain the hybrid network $(H)$ with 11 nodes, illustrated in Figure 2(e). Each node in this network has a different amount of connectedness, while high connectedness represents increased linkage between subgroups. In Figure 2(e), nodes 5, 6, and 7 have high connectedness since they connect to other subgroups within the network. These nodes are vital to maintaining connectivity. This diagram is used to capture large scale dynamics that other simpler models can not.

\subsection{Parameters and initial conditions}

In this section, we consider the $n$-Patch Model in Equations (1.4)-(1.6) with different network structures described in Section 2. In order to simulate the PPS dynamics on networks, we shall first carefully consider meaningful parameter ranges and initial conditions.

We will discuss the parameters and initial conditions used in different numerical simulations for these network structures. Initially, we take

$$
r^{(j)}=0.1
$$

In order to examine movement through hostile territory, we set the initial conditions of the system so that the first patch contains some prey, predator and subsidy and all other patches are empty,

$$
\begin{array}{lll}
x^{(1)}(0)=0.2, & x^{(j)}(0)=0, & \text { for } j \neq 1, \\
s^{(1)}(0)=0.2, & s^{(j)}(0)=0, & \text { for } j \neq 1, \\
y^{(1)}(0)=0.1, & y^{(j)}(0)=0, & \text { for } j \neq 1 .
\end{array}
$$

In Sections 3.2 and 3.3, we will examine the effect of the subsidy with different carrying capacity on the predator-preysubsidy model. We use the same input rate of prey stated in (2.1), and the same initial conditions stated in (2.2) in Section 3.2. However, we will perform the simulation with 100 values of input rate of subsidy. The value of $i^{(j)}$ starts from zero, increments by 0.01 , and ends at 1 . Also, we will perform the simulation with 250 values of input rate of subsidy. The value of $i^{(j)}$ is chosen from zero, increments by 0.002 , and ends at 0.5

\begin{tabular}{|c|c|}
\hline Case 1 & $\begin{aligned} r^{(1)}(0)=0.2, & i^{(1)}(0)=0.1 \\
r^{(j)}(0)=0 & \text { for } j \neq 1 \\
i^{(j)}(0)=0 & \text { for } j \neq 1\end{aligned}$ \\
\hline Case 2 & $\begin{aligned} r^{(1)}(0)=0.2, & i^{(2)}(0)=0.1 \\
r^{(j)}(0)=0 & \text { for } j \neq 1 \\
i^{(j)}(0)=0 & \text { for } j \neq 2\end{aligned}$ \\
\hline Case 3 & $\begin{array}{c}r^{(1)}(0)=0.2, \quad i^{(1)}(0)=0 \\
r^{(j)}(0)=0 \quad \text { for } j \neq 1 \\
i^{(j)}(0)=0.1 \quad \text { for } j \neq 1\end{array}$ \\
\hline
\end{tabular}
in Section 3.3. This allows us to create bifurcation diagrams.
Table 2: The data for the growth rate of the prey and the input rate of the subsidy for Case 1, 2, and 3 for all networks used.

Afterwards, in Sections 3.4-3.7, we examine the dynamics of system with different combinations of predator, prey and subsidy in order to better understand the role of network structure on PPS dynamics. We choose the following four cases to simulate all the bifurcation diagrams.

- Case 1: The prey and subsidy only exist in the $1^{\text {st }}$ patch. The initial condition is that some prey and subsides exist in the $1^{\text {st }}$ patch and some predator exist in the $6^{\text {th }}$ patch.

- Case 2: The prey only exists in the $1^{\text {st }}$ patch and the subsidy only exists in the $2^{\text {nd }}$ patch. The initial condition is that some prey exist in the $1^{\text {st }}$ patch, some subsidies exist in the $2^{\text {nd }}$ patch and some predators exist in the $6^{\text {th }}$ patch.

- Case 3: The prey only exists in the $1^{s t}$ patch and the subsidy only exists in all other patches. The initial condition is that some prey exist in the $1^{\text {st }}$ patch, some subsidies exist in all other patches and some predators exist in the $6^{\text {th }}$ patch.

- Case 4: About half of the patches contain the prey and about half of the patches contain the subsidy. The initial condition is that some prey exist in the first half number of patches, some subsidies exist in the last half number of patches, and some predators exist in the $6^{\text {th }}$ patch.

The data for the growth rate of the prey and the input rate of subsidy, and of the initial conditions for these four cases, are summarized in Tables $2-5$. We set the growth rate of the prey $\left(r^{(j)}=0.2\right)$ larger than the input rate of the subsidy $\left(i^{(j)}=0.1\right)$.

In Sections 3.4-3.7 we vary carrying capacity of the prey versus the predator migration rate, predator mortality rate versus predator migration rate, the proportion of predators surviving migration versus the predator migration rate, and the subsidy input rate versus the predator migration rate. To study each of these interactions, we need to fix a maximum value for the predator migration rate, $\alpha$, in order for the results to make biological sense. We can obtain all possible travel paths for this network. We can set a value between 0 and 1 for $\alpha^{(j, l)}$ if node $j$ and $l$ share an edge. In practice, we will fix $\alpha^{(j, l)}=\alpha$, a constant, for $0<\alpha<1$. Otherwise $\alpha^{(j, l)}$ is zero because there is no direct movement among these patches. For example, for $T$, both of 
Table 3: Initial conditions of the predator, prey and subsidy of Case 1, 2, and 3 for all networks used.

\begin{tabular}{|l|lcc|}
\hline \multirow{4}{*}{ Case 1 } & $x^{(1)}(0)=0.2$, & $x^{(j)}(0)=0$ & for $j \neq 1$ \\
& $s^{(1)}(0)=0.2$, & $s^{(j)}(0)=0$ & for $j \neq 1$ \\
& $y^{(6)}(0)=0.1$, & $y^{(j)}(0)=0$ & for $j \neq 6$ \\
\hline \multirow{5}{*}{ Case 2 } & $x^{(1)}(0)=0.2$, & $x^{(j)}(0)=0$ & for $j \neq 1$ \\
& $s^{(2)}(0)=0.2$, & $s^{(j)}(0)=0$ & for $j \neq 2$ \\
& $y^{(6)}(0)=0.1$, & $y^{(j)}(0)=0$ & for $j \neq 6$ \\
\hline \multirow{3}{*}{ Case 3 } & $x^{(1)}(0)=0.2$, & $x^{(j)}(0)=0$ & for $j \neq 1$ \\
& $s^{(1)}(0)=0, \quad s^{(j)}(0)=0.2$ & for $j \neq 1$ \\
& $y^{(6)}(0)=0.1$, & $y^{(j)}(0)=0$ & for $j \neq 6$ \\
\hline
\end{tabular}

Table 4: Growth rate of the prey and the input rate of the subsidy of Case 4 for all networks.

\begin{tabular}{|c|lcc|}
\hline \multirow{2}{*}{ 7-Patch } & $r^{(j)}(0)=0.2, \quad i^{(j)}(0)=0$ & for $j=1, \ldots, 4$ \\
& $r^{(j)}(0)=0, \quad i^{(j)}(0)=0$ & for $j=5,6,7$ \\
\hline 10-Patch & $r^{(j)}(0)=0.2, \quad i^{(j)}(0)=0$ & for $j=1, \ldots, 5$ \\
& $r^{(j)}(0)=0, \quad i^{(j)}(0)=0 \quad$ for $j=6, \ldots, 10$ \\
\hline 11-Patch & $r^{(j)}(0)=0.2, \quad i^{(j)}(0)=0 \quad$ for $j=1, \ldots, 5$ \\
& $r^{(j)}(0)=0, \quad i^{(j)}(0)=0 \quad$ for $j=6, \ldots, 11$ \\
\hline
\end{tabular}

Table 5: Initial conditions of the predator, prey, and subsidy of Case 4 for all networks.

\begin{tabular}{|c|cc|}
\hline \multirow{5}{*}{ 7-Patch } & $x^{(j)}(0)=0.2, \quad s^{(j)}(0)=0$ for $j=1, \ldots, 4$ \\
& $x^{(j)}(0)=0, \quad s^{(j)}(0)=0.2$ for $j=5,6,7$ \\
& $y^{(6)}(0)=0.1, \quad y^{(j)}(0)=0$ for $j \neq 6$ \\
\hline 10-Patch & $x^{(j)}(0)=0.2, \quad s^{(j)}(0)=0$ for $j=1, \ldots, 5$ \\
& $x^{(j)}(0)=0, \quad s^{(j)}(0)=0.2 \quad$ for $j=6, \ldots, 10$ \\
& $y^{(6)}(0)=0.1, \quad y^{(j)}(0)=0 \quad$ for $j \neq 6$ \\
11-Patch & $x^{(j)}(0)=0.2, \quad s^{(j)}(0)=0$ for $j=1, \ldots, 5$ \\
& $x^{(j)}(0)=0, \quad s^{(j)}(0)=0.2 \quad$ for $j=6, \ldots, 11$ \\
& $y^{(6)}(0)=0.1, \quad y^{(j)}(0)=0$ for $j \neq 6$
\end{tabular}

Table 6: The range of predator migration rates used in simulations, and the representative fixed value $\alpha=\bar{\alpha}$ for each network structure considered.

\begin{tabular}{|c|c|c|}
\hline Network & Range of $\alpha$ & $\bar{\alpha}$ \\
\hline Dendritic 7-Patch $(T)$ & {$[0,1 / 3]$} & 0.02 \\
\hline Linear 7-Patch $\left(P_{7}\right)$ & {$[0,1 / 2]$} & 0.02 \\
\hline Ring 10-Patch $\left(\mathrm{Ci}_{10}(1,2)\right)$ & {$[0,1 / 4]$} & 0.01 \\
\hline Complete Connected 10-Patch $\left(K_{10}\right)$ & {$[0,1 / 9]$} & 0.005 \\
\hline Hybrid Network $(H)$ & {$[0,1 / 4]$} & 0.02 \\
\hline
\end{tabular}

the $2^{\text {nd }}$ and $5^{\text {th }}$ patches have 3 neighbours each, the maximum number of neighbours in $T$. Therefore, the maximum $\alpha$-value we can set is $1 / 3$. The range of $\alpha$ values for different networks are summarized in Table 6 . There will be some cases where we fix $\alpha$ and vary other parameters, and the representative fixed values of $\alpha$ are also given in Table 6 .

In Sections 3.4-3.7, we will examine the effect of network structure on the predator-prey-subsidy model. We will examine the effect of the rate of predator's travel between patches, $\alpha^{(j, l)}$, with different values of carrying capacity, $k$, survival rate of predator's inter-patch migration, $\lambda^{(j, l)}$, and predator mortality rate, $\delta^{(j)}$ and input rate of subsidy, $i^{(j)}$. Therefore, we will use a range of $\alpha$ values stated in Table 6 to obtain bifurcation diagrams for each network. For simulations where we vary other parameters than $\alpha$, we shall fix $\alpha=\bar{\alpha}$ as indicated in Table 6 .

In Sections 3.2-3.5 and 3.7, we also need to fix a value for $\lambda^{(j, l)}$ for the time-series and all bifurcation diagrams except $\lambda-\alpha$ bifurcation diagrams. We set a fixed value between 0 and 1 for $\lambda^{(j, l)}$ if node $j$ and $l$ share an edge to represent the predator proportion which survive in any two nearby patches. In practice, $\lambda^{(j, l)}=\lambda$, a constant, in all numerical simulations, where $0<\lambda \leq 1$. The case of $\lambda=1$ represents all predators surviving the migration, and this is what we shall use for those bifurcation diagrams which do not vary in $\lambda$.

In Section 3.6, we will examine the effect of $\alpha^{(j, l)}$, with different values of survival rate of the predator's inter-patch migration, $\lambda^{(j, l)}$. Therefore, we will use a range of $\lambda$ values between 0 and 1 to achieve bifurcation diagrams for each network.

\section{Numerical Results}

\subsection{Numerical approach}

We considered the $n$-Patch Model in Equations (1.4)-(1.6) with different network structures as a high-order dynamical system. All the numerical simulations have been carried out using MATLAB's ode45 solver, which uses Runge-Kutta (4,5) [19]. We use the fixed value of $\alpha$ stated in Table 6, but different carrying capacity values $(k)$ and the input rate of the subsidy $\left(i^{(j)}\right)$ to achieve four different outcomes. The outcomes for the predator-prey-subsidy model are qualitatively similar for these five networks. In this section, we choose the hybrid network $H$ with 11 patches as an example. Figure 3 shows these outcomes for the hybrid network including (a) a prey-free equilibrium, (b) a predator-free equilibrium, (c) an equilibrium in which the predator, prey, and subsidy coexist, and (d) coexistence under stable limit cycles. In our simulation, we consider the system 

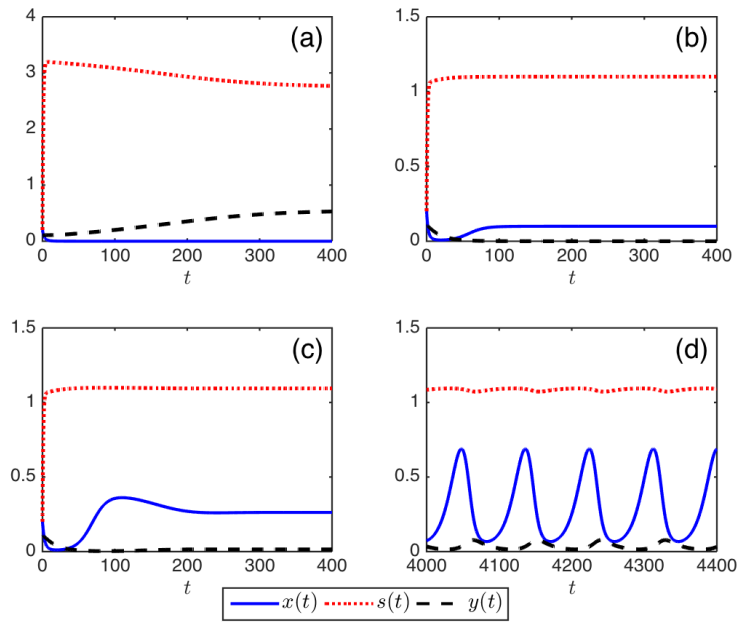

Figure 3: The dynamics of the PPS on hybrid network $(H)$ when all parameters are constant. In (a) the prey becomes extinct, the subsidy amount approaches its natural value, and the predator population size approaches a positive equilibrium value $\left(k=0.1\right.$ and $\left.i^{(j)}=0.3\right)$. In (b) the prey population size approaches a positive equilibrium value, the subsidy amount approaches its natural value, and the predator becomes extinct $\left(k=0.1\right.$ and $\left.i^{(j)}=0.1\right)$. In (c) the predator and prey population sizes approach positive equilibrium values and the subsidy amount approaches its natural value $\left(k=0.4\right.$ and $\left.i^{(j)}=0.1\right)$. In $(\mathrm{d})$ the predator, prey, and subsidy all persist in a stable limit cycle $\left(k=2.4\right.$ and $\left.i^{(j)}=0.1\right)$. The parameters are $r^{(j)}=0.1, \theta=5, h=1, \gamma^{(j)}=1, \psi=5, \epsilon=0.1, \eta=0.1$ and $\delta^{(j)}=0.1$ for $j \in[1,11]$. The initial conditions are $x^{(1)}(0)=0.2, s^{(1)}(0)=0.2$, $y^{(1)}(0)=0.1, x^{(j)}(0)=0, s^{(j)}(0)=0$ and $y^{(j)}(0)=0$ for $j \neq 1$.

as prey-free or predator-free when the sum of $x(t)$ or $y(t)$ in all patches is less than $10^{-5}$. (Note: In Figure 3(c), the total population of the predator is above $10^{-5}$.) Our goal is to distinguish situations where these different outcomes occur, and to understand the influence of both the parameter values and the network structures on these outcomes.

\subsection{Effect Of Subsidy On Predator And Prey}

We first examine the effects of introducing subsidies into the predator-prey system in order to demonstrate the importance of the subsidy when it is sufficiently influential in the system. In this section, we choose a perfect binary tree network with 7 patches as an example since the qualitative behaviour of the effect of the subsidy on the predator and prey are similar for other networks.

Figure 4 shows the effect of increasing the input rate of subsidy, $i^{(j)}$, on the population size of the prey and predator for $T$. The plots of $x(t)$ and $y(t)$ in Figure 4 represent the sum of the population of the prey and predator in all patches. An equilibrium is approached and is displayed when the solution tends to a single value for large time. If the solutions oscillate, we can show minimum and maximum values of the resulting limit cycle. We use $k=0.1,0.4,2.4$ and 5 in (a), (b), (c), and (d), respectively, in Figure 4.

In Figure 4(a), when $i^{(j)}<0.22$, the system is in a predatorfree equilibrium; when $i^{(j)}$ increases from 0.22 to 0.27 , there is a system where predator and prey coexist; when $i^{(j)}$ increases from 0.27 onwards, the system goes to a prey-free equilibrium.
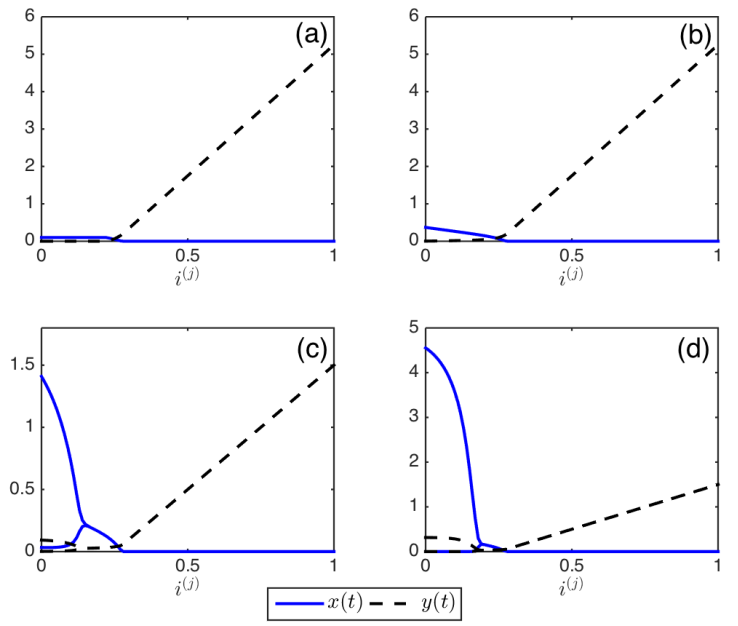

Figure 4: The effect of the subsidy input rate $i^{(j)}$, on the stable equilibria ((a) and (b)) and limit cycle ((c) and (d)) of the dynamics of (1.4)-(1.6) on $T$, with the maximum and minimum values of the stable dynamics plotted. In (a), (b), (c) and (d) we use $k=0.1,0.4,2.4$ and 5 respectively. The parameters used are $r^{(j)}=0.1, \theta=5, h=1, \gamma^{(j)}=1, \psi=5, \epsilon=0.1, \eta=0.1$ and $\delta^{(j)}=0.1$ for $j \in[1,7]$.

When the uninterrupted system has a stable equilibrium (in Figures 4(a) and (b)), the system approaches an equilibrium when no subsidy is present. As $i^{(j)}$ starts increasing from zero, there is more subsidy, meaning more food available for the predator. The predator population size increases and the prey population size decreases. When the subsidy input rate is sufficiently large to cause extinction of the prey, the predator population size at equilibrium increases faster than the subsidy input rate. When the uninterrupted system has a limit cycle (in Figures 4(c) and (d)), increasing $i^{(j)}$ stabilises the dynamics. When $i^{(j)}$ is sufficiently large, there is no prey in the system, and there is an increased sensitivity again of the predator equilibrium value to the subsidy input rate. We use larger $k$ in Figure 4(d) compared to Figure 4(c). We achieve a larger limit cycle in Figure 4(d) due to the increased carrying capacity.

\subsection{Subsidy Input Rate Versus Prey's Carrying Capacity}

To examine the subsidy effect with more carrying capacity values, we study the dynamics of the predator-prey-subsidy model for all networks with a set of the carrying capacity $(k)$ and subsidy input rates $\left(i^{(j)}\right)$. There is no qualitative difference in plots obtained for all networks demonstrated in Figure 2, so we choose one network, $K_{10}$, as an example. This was previously studied in [23] for one and two-patch cases. Therefore, under more complicated networks, we get similar results.

In the numerical simulation, we define the system as preyfree or predator-free when the sum of $x(t)$ or $y(t)$ in all patches is less than $10^{-5}$. We also define a non-equilibrium region, usually a limit cycle region, when the absolute value of the difference of maximum and minimum of $x(t)$ or $y(t)$ is larger than 0.1 . Figure 5 shows the outcome of the predator-prey-subsidy model for different subsidy input rates and different values of the carrying capacity for the complete graph $K_{10}$. The dynamics in the bifurcation diagram are those of a predator-free equi- 


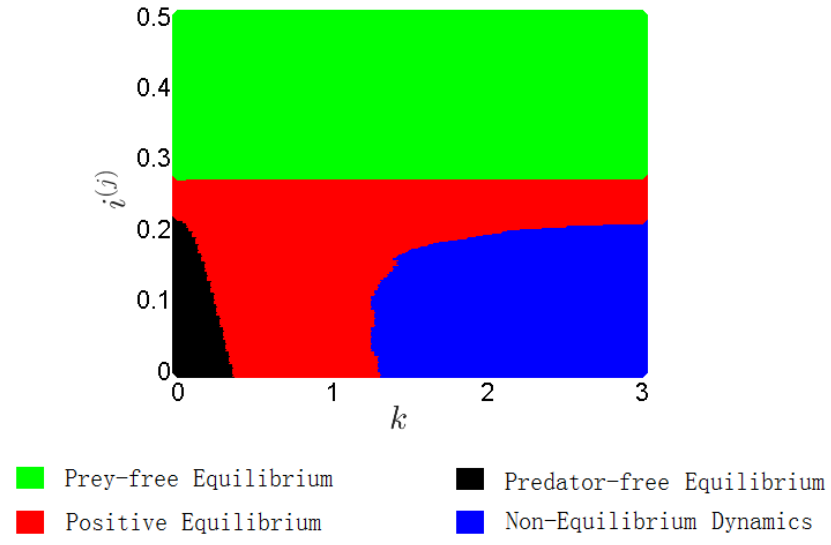

Figure 5: The outcome of the PPS model on $K_{10}$ for different subsidy input rates $\left(i^{(j)}\right)$ and different values of the carrying capacity $(k)$. The parameters used are $r^{(j)}=0.1, \theta=5, h=1, \gamma^{(j)}=1, \psi=5, \epsilon=0.1, \eta=0.1$ and $\delta^{(j)}=0.1$.

librium in the lower-left region (black), a prey-free equilibrium in the upper region (green), a positive equilibrium in the central region (red), or non-equilibrium dynamics (including, for example, limit cycles) involving predator, prey, and subsidy in the lower-right region (blue). Figure 4 in the previous section supports this conjecture (Figure 5).

In Figure 5, the prey becomes extinct when $i^{(j)}$ is larger than 0.28 for any possible $k$ between 0 and 3. When $0.22<i^{(j)}<$ 0.28 , the system will achieve a positive equilibrium. When $i^{(j)}<0.22$, we have a predator-free equilibrium, a positive equilibrium or a stable limit cycle for different values of $k$. Therefore, we will examine the effect of $\alpha^{(j, l)}$ on the system in Sections 3.4-3.7 with only one fixed value of $i^{(j)}$ less than 0.22 . The positive equilibrium region exists when $k>0.4$ and is stable only for $k<1.3$ for $i^{(j)}<0.22$. When $k>1.3$, the system moves to a non-equilibrium region (including dynamics such as limit cycles). The destabilization of the positive equilibrium because of a increase in the carrying capacity is normally referred to as the paradox of enrichment $[23,20,5,6,25,28]$.

\subsection{Prey Carrying Capacity Versus Predator Migration Rate}

We now examine the effect of the rate of predator's travel between patches, $\alpha^{(j, l)}$, with different values of carrying capacity for the prey, $k$. We want to examine the dynamics of system with different combinations of predators, prey and subsidies. For each case, we use different combinations of the growth rates of the prey, input rates of the subsidy, and initial conditions of the system, stated in Tables 2-5. Also, we fix the input subsidy rate as $i=0.1$.

As a demonstration, the dynamics corresponding to the four cases of the initial condition combinations for the perfect binary tree network $(T)$ are shown in Figure 6. For Case 1 in Figure 6 , when $\alpha<0.08$, the system moves to a non-equilibrium region (such as a limit cycle region), a predator-free equilibrium region, or a positive equilibrium region with different $k$ values. We also have a higher probability of having a stable limit cycle as $k$ increases. For $\alpha>0.08$, the limit cycle region disappears, and the system can only move to a positive equilibrium
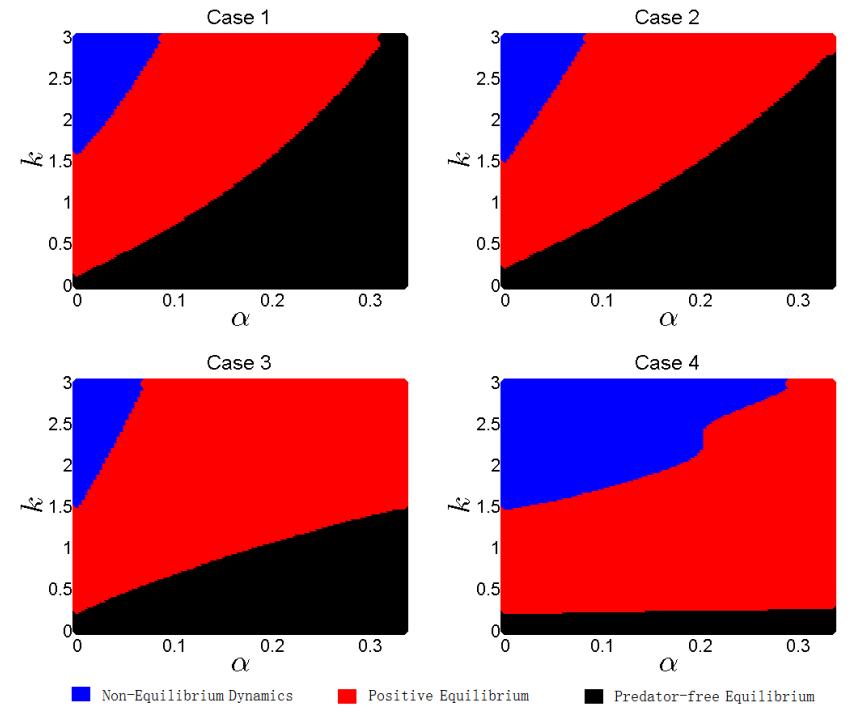

Figure 6: Outcome of the PPS model on $T$ for different values of the carrying capacity $(k)$ and different rates at which the predator travels $\left(\alpha^{(j, l)}\right)$ for various initial conditions (Case 1-4). The parameters used are $\theta=5, h=1, \gamma^{(j)}=1$, $\psi=5, \epsilon=0.1, \eta=0.1$ and $\delta^{(j)}=0.1$ for $j \in[1,7]$.

or a predator-free equilibrium with different $k$ values. When a larger proportion of predators move to other patches, the predator population size will decrease since there is neither prey nor subsidy in the other patches. The simulation result for Case 1 shows that as $\alpha$ increases, we actually have a higher possibility of the predator becoming extinct. This may be due to the predators spending too much time moving through regions which are poor in food sources.

Figure 6 shows the outcome for the other three cases of initial conditions as well. These figures contain the same three dynamics, but with different proportions. In Case 2, the predator-free region is slightly smaller compared with Case 1 . This is reasonable because we have an extra patch, the second patch, that has the subsidy. The predators have more food to consume which increases their survival rate. The predator-free region in Case 3 is much smaller than it in Case 1, because we have subsidies not only in one patch, but in six patches in Case 3, which would lower the predator extinction probability. In general, for Case $1-3$, we have higher possibility of the predator going extinct as $\alpha$ increases. In Case 4, we have four patches that only contain the prey and the other three patches only contain the subsidies. Because of sufficient food, the predators have a much lower possibility of going extinct compared with the other three cases. The predator-free region is very small, and only exists when $k<0.25$. There is a larger region of the limit cycle in Case 4 compared with the other cases because we have more food sources available. We also have a larger positive equilibrium region and a smaller limit cycle region as $\alpha$ increases, thus in this case, more predator migration stabilizes the system.

Similarly, we examine the dynamics for these four cases for the other four networks. The results for Case 1-3 are qualitatively similar for all networks. Therefore, we choose one of them, Case 2, as an example to demonstrate. The outcome of 
(a)

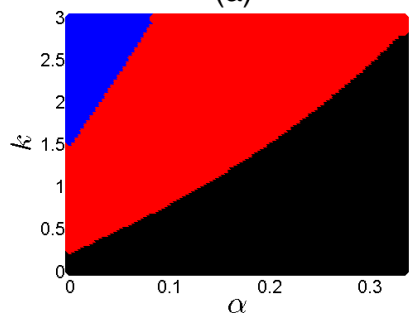

(c)

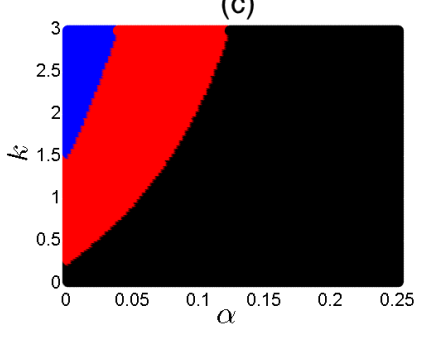

口

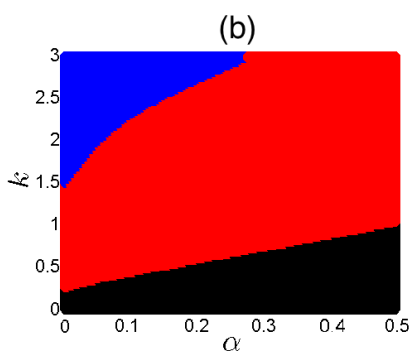

(d)

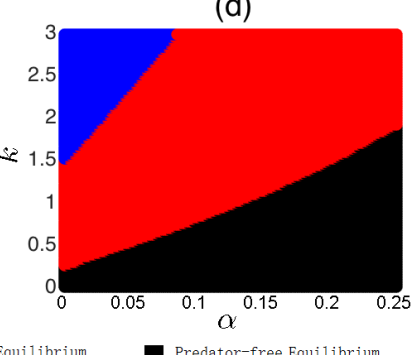

Figure 7: The Case 2 outcome of the PPS model on different networks for different values of the carrying capacity $(k)$ and different rates at which the predator travels $\left(\alpha^{(j, l)}\right)$. Here, (a) $T$, (b) $P_{7}$, (c) $C i_{10}(1,2)$, and (d) $H$. The parameters used are $\theta=5, h=1, \gamma^{(j)}=1, \psi=5, \epsilon=0.1, \eta=0.1$ and $\delta^{(j)}=0.1$.

(a)

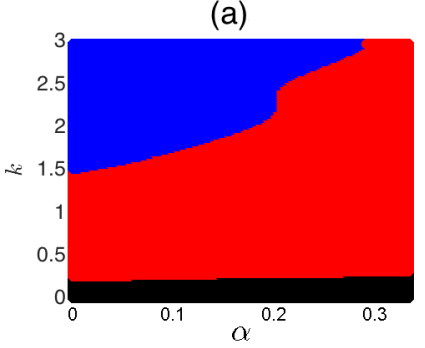

(c)
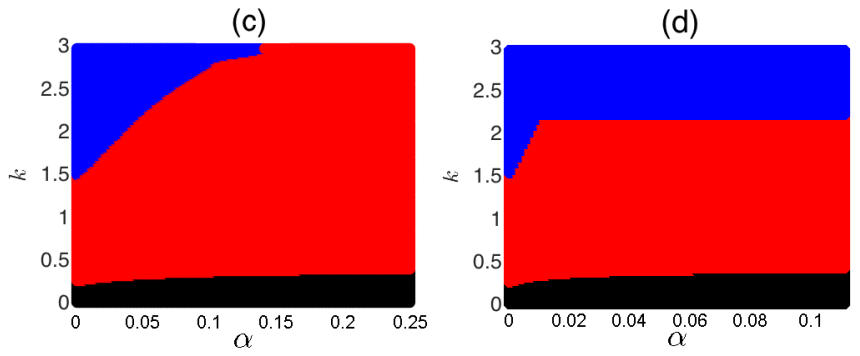

(e)

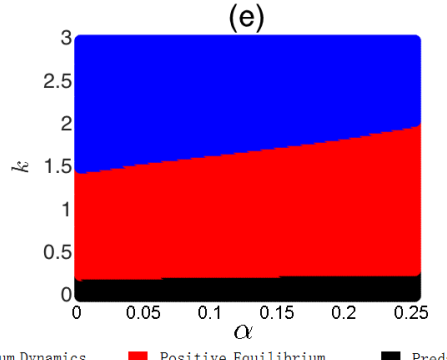

- Non-Equi1ibrium Dynamics

Positive Equilibrium

Figure 8: The Case 4 outcome of the PPS model on different networks for different values of the carrying capacity $(k)$ and different rates at which the predator travels $\left(\alpha^{(j, l)}\right)$. Here, (a) $T$, (b) $P_{7}$, (c) $C i_{10}(1,2)$, (d) $K_{10}$, and (e) $H$. The parameters used are $\theta=5, h=1, \gamma^{(j)}=1, \psi=5, \epsilon=0.1, \eta=0.1$ and $\delta^{(j)}=0.1$.
$K_{10}$ is qualitatively similar as that of $C i_{10}(1,2)$, so Figure 7 only shows the outcome of Case 2 for $T, P_{7}, C i_{10}(1,2)$, and $H$.

In Figure 7, the non-equilibrium region in (b) is slightly larger than in (a) because of the increase of the food source, and the positive equilibrium region is relatively larger as well. However, the predator-free region is much smaller in (b) compared to (a). With regards to $C i_{10}(1,2)$ (Figure $7(\mathrm{c})$ ), the nonequilibrium and positive equilibrium regions are much smaller and the predator-free region is much larger than in Figure 7(a). The reason is that $C i_{10}(1,2)$ has three more patches compared with $T$, meaning that predators have more patches to move to, but the prey and subsidy are only in the first two patches. So, more movement will result in a higher probability of death for the Case 2 condition.

We also note that Figure 7(d) is similar to Figures 7(a) and (b) and is more favourable for the predator than Figure 7(c). Even though $H$ has the most patches, it combines behaviour from other networks, enabling it to have a similar non-equilibrium and positive equilibrium to $T$ and $P_{7}$. However, the predatorfree region for $H$ is slightly larger than that in $P_{7}$. Therefore, $P_{7}$ is the most favourable for coexistence in the given condition.

Next, Figure 8 shows the outcome of Case 4 for all the networks. In this case, half of the patches have prey and the other half have subsidies. Therefore, all patches contain food for the predator. For this case, more movement does not necessarily lead to a higher predator mortality rate, which is shown in Figure 8 . By extension, the predator-free region for Case 4 (Figure 8 ) is much smaller than that for Case 2 (Figure 7).

The outcomes for $T$ and $P_{7}$ are similar in Figure 8. For $C i_{10}(1,2)$, the positive equilibrium region is twice as large as that of $P_{7}$ for $\alpha \in[0,0.25]$, while the non-equilibrium region is slightly smaller compared to that of $P_{7}$ in the same range of $\alpha$ values. The predator-free region for $P_{7}$ and $C i_{10}(1,2)$ are similar. This is reasonable because for Case 4 , more movement enables predators to seek out multiple food sources. Also, the non-equilibrium region for $K_{10}$ is larger than for $C i_{10}(1,2)$. For $H$, the distribution is similar compared with that of $K_{10}$, but the positive equilibrium and predator-free regions are slightly smaller. Therefore, $C i_{10}(1,2)$ is the most favourable for coexistence for the Case 4 condition.

\subsection{Predator Mortality Rate Versus Predator Migration Rate}

The effect of predators travelling between patches $\left(\alpha^{(j, l)}\right)$ with different mortality rates $\left(\delta^{(j)}\right)$ is now considered. The growth rate of the prey, input rate of the subsidy, and initial conditions of all species are shown in Tables 2-5. In this section, we assume there is no death due to migration, and we decide to choose a large and a small value of the carrying capacity to examine the effect of predator mortality rate. If we include deaths due to migration, we have a slight shift in the bifurcation diagrams when migration deaths are also incorporated, although there is no drastic qualitative change in behavior unless the proportion of the population surviving migration is very small. So, considering $\lambda=1$ or $\lambda=0.5$ (half of the population dies in migration) does not give drastically different results in a qualitative sense for most cases, although there are of course quantitative differences, particularly with where the various bifurcation 

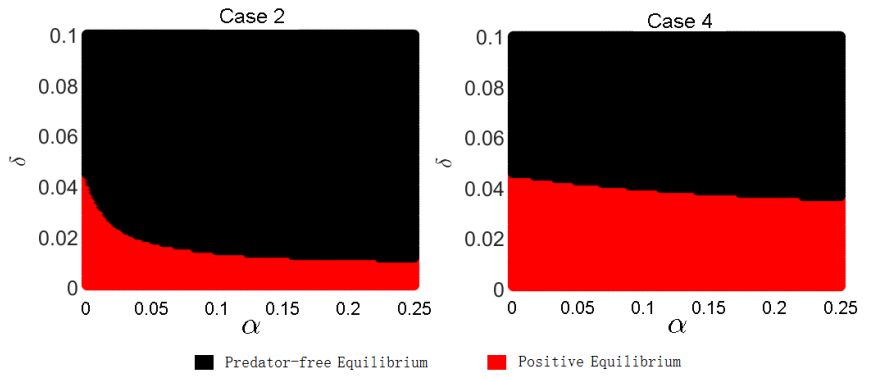

Figure 9: The Case 2 and Case 4 outcome of the PPS model on $H$ for different mortality rates of predators $\left(\delta^{(j)}\right)$ and different rates at which the predator travels $\left(\alpha^{(j, l)}\right)$. The parameters are $\boldsymbol{k}=\mathbf{0 . 1}, \theta=5, h=1, \gamma^{(j)}=1, \psi=5, \epsilon=0.1$, and $\eta=0.1$.
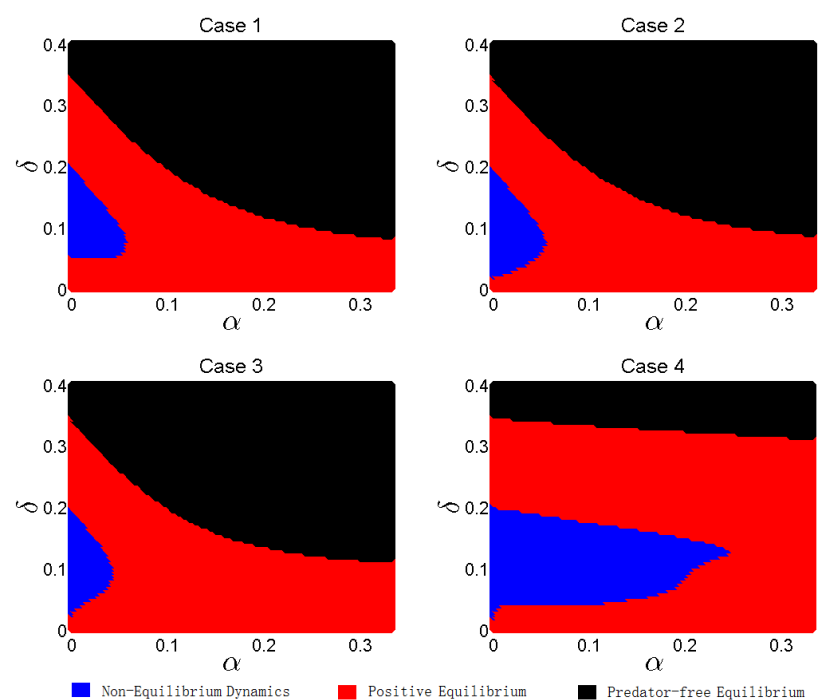

Figure 10: Outcome of the PPS model on $T$ for different mortality rates of predators $\left(\delta^{(j)}\right)$ and different rates at which the predator travels $\left(\alpha^{(j, l)}\right)$ for various initial conditions (Case 1-4). The parameters are $\boldsymbol{k}=\mathbf{2 . 4}, \theta=5, h=1$, $\gamma^{(j)}=1, \psi=5, \epsilon=0.1$ and $\eta=0.1$.

boundaries lie in the individual bifurcation diagrams. In the next section, Section 3.6, we see the influence of the migration mortality parameter $(\lambda)$ on the dynamics given fixed mortality rate within the patch $(\delta)$.

Firstly, we choose $k=0.1$ and the Case 2 conditions to obtain the $\delta-\alpha$ bifurcation diagram for the hybrid network, shown in the left diagram in Figure 9. When $\delta^{(j)}>0.045$, the system reaches a predator-free region for all $\alpha$. When $\delta^{(j)}<0.045$, we have an increasing predator-free region and decreasing positive equilibrium region as $\alpha$ increases. With a minimal carrying capacity value $(k=0.1)$, predators can only survive with very low death rates. Then we use the Case 4 conditions to obtain the right diagram in Figure 9, and find that the threshold for a predator-free zone still does not exceed 0.045 . The predatorfree region for Case 4 is smaller than it is in Case 2 because predators have more food sources leading to a higher survival rate. The outcomes for other networks are qualitatively similar to that for $H$, so there is no strong correlation between network structure on the bifurcation results when $k$ is small. (a)
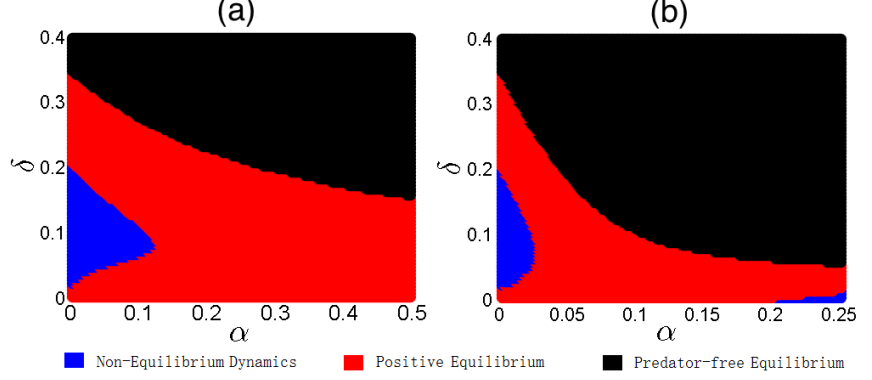

Figure 11: The Case 2 outcome of the PPS model on different networks for different mortality rate of predators $\left(\delta^{(j)}\right)$ and different rates at which the predator travels $\left(\alpha^{(j, l)}\right)$. Here, (a) $P_{7}$ and (b) $C i_{10}(1,2)$. The parameters are $\boldsymbol{k}=\mathbf{2 . 4}$, $\theta=5, h=1, \gamma^{(j)}=1, \psi=5, \epsilon=0.1$, and $\eta=0.1$.

(a)

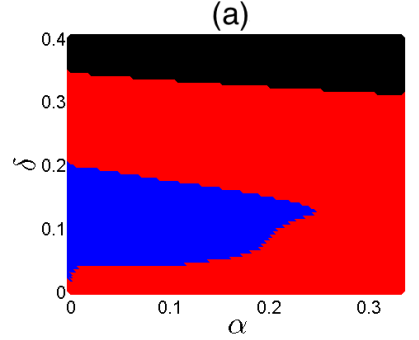

- Non-Equilibrium Dynamics $\quad$ Positive Equilibrium

(c)

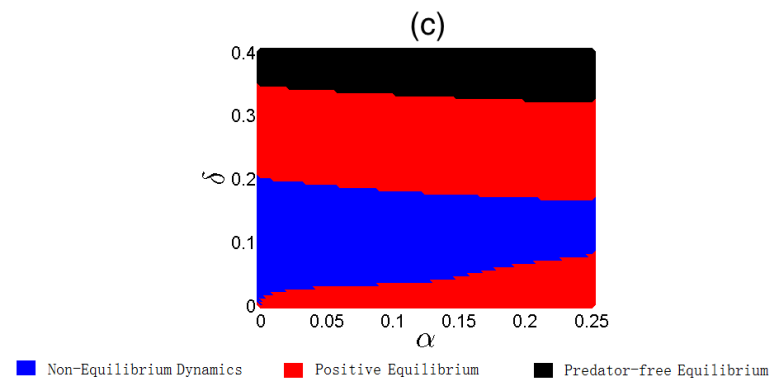

(b)

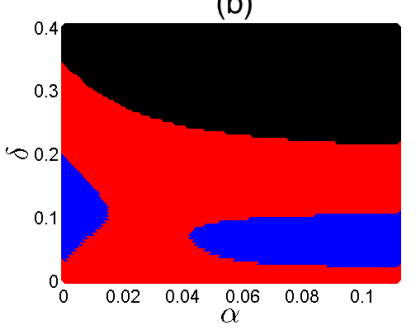

Figure 12: The Case 4 outcome of the PPS model on different networks for different mortality rates of predators $\left(\delta^{(j)}\right)$ and different rates at which the predator travels $\left(\alpha^{(j, l)}\right)$. Here, (a) $T$, (b) $K_{10}$, and (c) $H$. The parameters are $\boldsymbol{k}=\mathbf{2 . 4}$, $\theta=5, h=1, \gamma^{(j)}=1, \psi=5, \epsilon=0.1$, and $\eta=0.1$.

We choose the perfect binary tree network $T$ and set a larger value of $k(k=2.4)$ to obtain a non-equilibrium (i.e. limit cycle) region for certain $\delta$ and $\alpha$ values, shown in Figure 10. With a larger carrying capacity, the predators survive more easily even with larger mortality rates. The mortality rate threshold increases to 0.36 for these four cases. The system moves to a non-equilibrium region when the death rate is between 0.05 and 0.2 and $\alpha$ is smaller than about 0.05 for Cases $1-3$. For Case 4 , the non-equilibrium region is much larger compared with the other three cases because of more food. When the mortality rate is 0.1 , the system moves to a non-equilibrium region when $\alpha$ is small and moves to a stable positive equilibrium when $\alpha$ is larger. We chose $\delta^{(j)}$ to be 0.1 when examining the effect of $\alpha$ in other sections for this reason.

Finally, we choose the conditions of Case 2 and Case 4 with $k=2.4$ to obtain the $\delta-\alpha$ bifurcation diagrams for different networks, shown in Figures 11 and 12 respectively. For the Case 2 condition, the outcome of $T, P_{7}$ and $H$ are qualitatively similar, 
so we choose one of the networks, $P_{7}$, as an example. Also, the outcome of $C i_{10}(1,2)$ and $K_{10}$ are qualitatively similar, so we choose $C i_{10}(1,2)$ as an example. In Figure 11 , the mortality rate threshold value of $P_{7}$ and $C i_{10}(1,2)$ has increased to 0.36 , so the predators are more likely to survive with larger death rates with the large carrying capacity. In Figure 11(a), when $\delta^{(j)}$ is smaller than 0.16 , the system moves from the non-equilibrium region to the positive equilibrium region as $\alpha$ increases. Therefore, a higher proportion of movement will stabilize the system when $\delta^{(j)}$ is smaller than 0.16 . In Figure $11(\mathrm{~b})$, when $\delta^{(j)}$ is very small (less than 0.02), the system moves from the positive equilibrium region to the non-equilibrium region as $\alpha$ increases. Also, the predator-free region in Figure 11(b) is larger than it is in Figure 11(a). Because food is present in insufficient quantities, more movement will lead to a higher mortality rate. Therefore, $P_{7}$ is more suitable for coexistence than $C i_{10}(1,2)$.

For the Case 4 conditions, the outcome of $T, P_{7}$ and $C i_{10}(1,2)$ are qualitatively similar, so we choose one of the networks, $T$, as an example. Figure 12 shows the $\delta-\alpha$ diagrams for $T, K_{10}$ and $H$. In Figure 12, the threshold value of $\delta^{(j)}$ is still the same at 0.36 . However, the positive equilibrium and non-equilibrium regions are much larger, with the predatorfree region significantly reduced compared with the outcomes of the Case 2 condition shown in Figure 11. In Figure 12(a), when $\delta^{(j)}$ is smaller than 0.32 , the system will become more stable as $\alpha$ increases. In Figure 12(b), the predator-free region is larger the ones in Figures 12(a) and (c). Also, we note that for $\delta^{(j)}$ values from 0.04 to 0.1 , the system moves from the nonequilibrium region to the positive equilibrium and back to the non-equilibrium region again as $\alpha$ increases. Figure 12(c) is similar to Figure 12(a) if we look at $\alpha$ from 0 to 0.25 . Overall, both $T$ and $H$ are the most suitable for coexistence.

\subsection{Proportion Of Predators Surviving Migration Versus Predator Migration Rate}

In this section, we will examine the effects of predators travelling between patches $\left(\alpha^{(j, l)}\right)$ with different survival rates of inter-patch migration $\left(\lambda^{(j, l)}\right)$. The growth rate of prey, input rate of the subsidy, and the initial conditions of all species are shown in Tables 2-5.

First, we choose $k=0.4$ and the Case 3 conditions to obtain the $\lambda-\alpha$ bifurcation diagrams for $K_{10}$ and $H$, shown in Figure 13. The outcomes of $T, P_{7}, C i_{10}(1,2)$ and $K_{10}$ are not qualitatively different, so we choose one network, $K_{10}$, as an example. In Figure 13, we only have a positive equilibrium region for any $\lambda$ values when $\alpha$ is very small. As $\alpha$ increases, the system always moves to a predator-free region for the Case 3 condition. The positive equilibrium region in Figure 13(b) is four times larger than it is in Figure 13(a), and it increases slightly as the migration survival rate increases, which is reasonable behavior. $H$ is slightly better than $K_{10}$ in terms of permitting coexistence of predator and prey.

We next choose $k=0.4$ and the Case 4 conditions to obtain the $\lambda-\alpha$ bifurcation diagrams for $K_{10}$ and $H$, shown in Figure 14. The outcomes of $T, P_{7}, C i_{10}(1,2)$ and $K_{10}$ are qualitatively similar, so we choose $K_{10}$ to illustrate the result. Figure 14(a) (a)

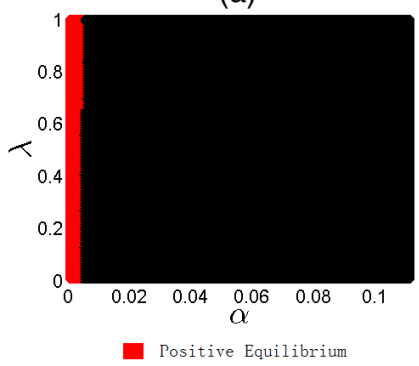

(b)

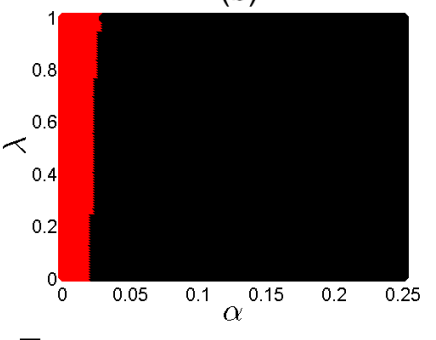

Predator-free Equilibrium
Figure 13: The Case 3 outcome of the PPS model on different networks for different survival rates of inter-patch migration of predators $\left(\lambda^{(j, l)}\right)$ and different rates at which the predator travels $\left(\alpha^{(j, l)}\right)$. Here, (a) $K_{10}$ and (b) $H$. The parameters are $\boldsymbol{k}=\mathbf{0 . 4}, \theta=5, h=1, \gamma^{(j)}=1, \psi=5, \epsilon=0.1, \eta=0.1$ and $\delta^{(j)}=0.1$. (a)

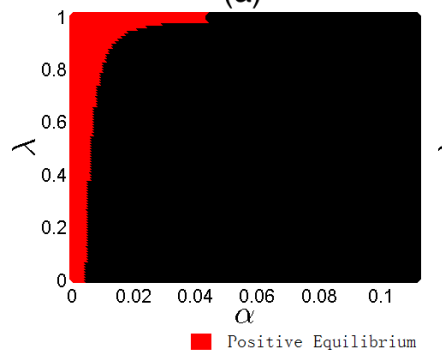

(b)

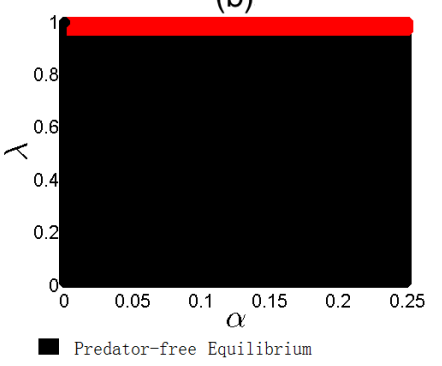

Figure 14: The Case 4 outcome of the PPS model on different networks for different survival rates of inter-patch migration of predators $\left(\lambda^{(j, l)}\right)$ and different rates at which the predator travels $\left(\alpha^{(j, l)}\right)$. Here, (a) $K_{10}$ and (b) $H$. The parameters are $\boldsymbol{k}=\mathbf{0 . 4}, \theta=5, h=1, \gamma^{(j)}=1, \psi=5, \epsilon=0.1, \eta=0.1$ and $\delta^{(j)}=0.1$.

for Case 4 is similar Figure 13(a), except the positive equilibrium region increases when $\lambda$ is large. However, in Figure 14(b), when $\alpha<0.01$, we have a predator-free region for any $\lambda$ values. When $\lambda>0.95$, our system moves from the predatorfree region to a positive equilibrium region as $\alpha$ is larger than 0.01. With Case 4 conditions, $H$ demonstrates that more movement will stabilise the system when the inter-patch migration survival rate of predators is larger than 0.95 .

We next choose $k=2.4$ and the Case 3 conditions to obtain the $\lambda-\alpha$ bifurcation diagrams for $T$ and $C i_{10}(1,2)$, shown in Figure 15 . The outcome of $T, P_{7}, H$ are qualitatively similar, so we choose one of the networks, $T$, as an example. Also, there is no qualitative difference between the outcome of $C i_{10}(1,2)$ and $K_{10}$, so we choose $C i_{10}(1,2)$ to demonstrate the results. For Case 3, the first patch has prey while the other patches have subsidies. In Figure 15(a), as $\alpha$ increases, the system moves from the non-equilibrium to the positive equilibrium and finally approaches the predator-free region, except when $\lambda$ is smaller than 0.95 . When $\lambda$ is larger than 0.95 , the system becomes more stable when $\alpha$ increases. However, in Figure 15(b), the system will eventually move to the predator-free region as $\alpha$ increases for all $\lambda$ values. Therefore, $T$ is the most favourable for coexistence for the given conditions.

Finally, we choose $k=2.4$ and the Case 4 conditions to obtain the $\lambda-\alpha$ bifurcation diagrams for all networks, shown in 
(a)

(a)

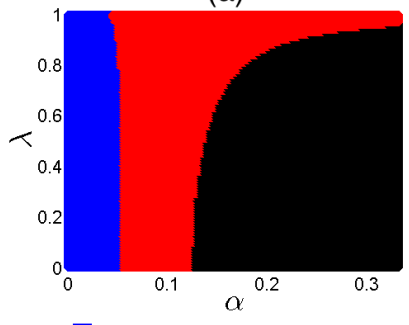

- Non-Equilibrium Dynamics (b)

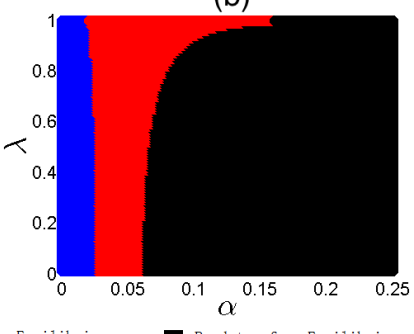

Figure 15: The Case 3 outcome of the PPS model on different networks for different survival rates of inter-patch migration of predators $\left(\lambda^{(j, l)}\right)$ and different rates at which the predator travels $\left(\alpha^{(j, l)}\right)$ for different networks. Here, (a) $T$ and (b) $C i_{10}(1,2)$. The parameters are $\boldsymbol{k}=\mathbf{2 . 4}, \theta=5, h=1, \gamma^{(j)}=1, \psi=5$, $\epsilon=0.1, \eta=0.1$ and $\delta^{(j)}=0.1$.

(a)

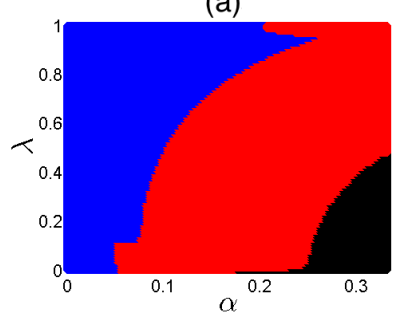

(c)
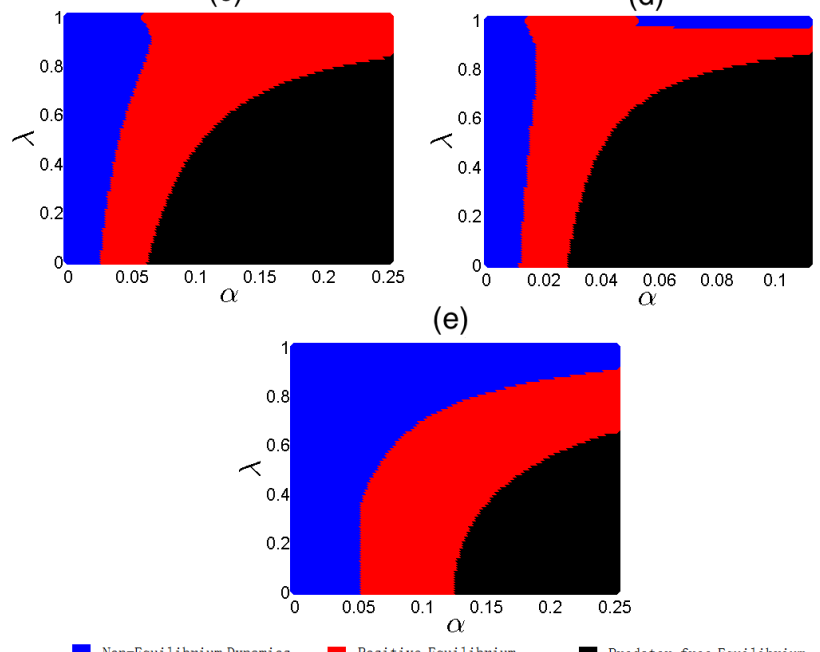

- Non-Equi1ibrium Dynamics $\square$ Positive Equilibrium

Figure 16: The Case 4 outcome of the PPS model on different networks for different survival rates of inter-patch migration of predators $\left(\lambda^{(j, l)}\right)$ and different rates at which the predator travels $\left(\alpha^{(j, l)}\right)$. Here, (a) $T$, (b) $P_{7}$, (c) $C i_{10}(1,2)$, (d) $K_{10}$, and (e) $H$. The parameters are $\boldsymbol{k}=\mathbf{2 . 4}, \theta=5, h=1, \gamma^{(j)}=1, \psi=5$, $\epsilon=0.1, \eta=0.1$ and $\delta^{(j)}=0.1$.

(b)

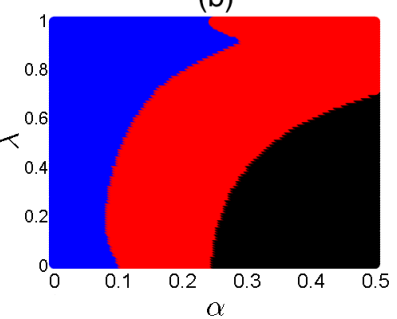

(d)

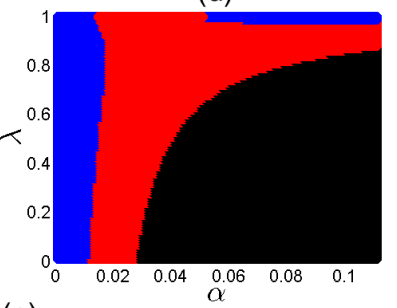

(e) 
(a)

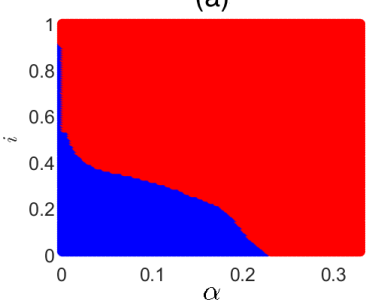

(c)
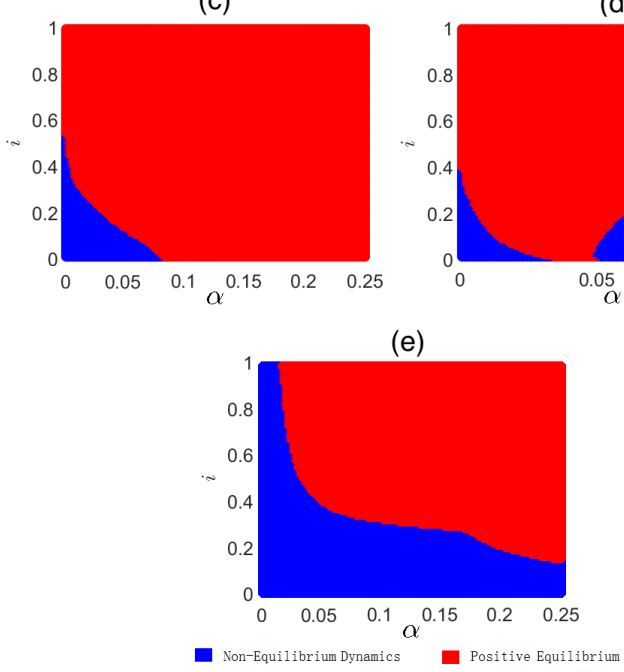

Figure 18: The Case 4 outcome of the PPS model on different networks for different subsidy input rates $\left(i^{(j)}\right)$ and different rates at which the predator travels $\left(\alpha^{(j, l)}\right)$. Here, (a) $T$, (b) $P_{7}$, (c) $C i_{10}(1,2)$, (d) $K_{10}$, and (e) $H$. The parameters are $k=\mathbf{2 . 4}, \theta=5, h=1, \gamma^{(j)}=1, \psi=5, \epsilon=0.1, \eta=0.1$ and $\delta^{(j)}=0.1$.

(d) have very large predator-free regions, while the predatorfree region of Figure 17(a) is much smaller. For Figure 17(b) and (e), when we have more predator movement, the system becomes more stable, but the predator goes extinct in Figures 17(a), (c), and (d). Overall, $H$ is the most favourable for coexistence for the given condition.

We choose $k=2.4$ and the Case 4 conditions to obtain the $i-\alpha$ bifurcation diagrams for all networks, shown in Figure 18. In general, as the subsidy input rate increases, the positive equilibrium replaces the limit cycles. For Case 4 with more food sources, the system becomes more stable due to higher predator migration rates in all diagrams except Figure 18(d). In Figure 18(d), when $i<0.4$, the system moves from non-equilibrium to equilibrium states, and finally back to non-equilibrium.

So, for $K_{10}$, we see that increasing $\alpha$ (for small enough $i$ ) acts to actually destabilise the dynamics, pushing the coexistence equilibrium into a limit cycle. This is akin to what happens when we increase $k$ and find a similar bifurcation (paradox of enrichment), hence in this case the additional movement gives dynamics which mirror the paradox of enrichment.

Note that we are familiar with no existing results in the predator-prey literature showing that increased movement induces limit cycles. Therefore, that increased movement results in something not unlike what we see in the paradox of enrichment is rather exciting.

We do not see this behavior in the other networks consid- ered, and therefore it seems as though such a destabilization of coexistence equilibria into limit cycles via movement will only occur for specific families of networks. Therefore, this result suggests that the underlying network structure can be highly important in determining predator-prey dynamics.

\section{Discussion}

We model predator-prey-subsidy systems, using arctic foxes, lemmings, and seal carcasses provided by polar bears as a motivating example. Our analysis is applicable to any other system which models predator-prey-subsidy ecosystems. We have used a $3 n$-dimensional systems of nonautonomous, nonlinear ODEs, developed by Nevai and Van Gorder in [23], to analyse predator-prey-subsidy dynamics on networks. This model was named the $n$-Patch Model. In the $n$-Patch Model, each patch in the Arctic is modelled by a spatially-separated patch in a heterogeneous environment. We investigate PPS dynamics on different networks because the network structures have some influence on population dynamics. Our objective is to examine the effect of including network structures on predator-preysubsidy dynamics of (1.4)-(1.6). We consider dynamics of $n$ Patch Model for four different cases, including patches with different growth rates of prey, input rates of subsidy, and initial conditions. Also, we consider the role that the carrying capacity $(k)$, predator migration rate $\left(\alpha^{(j, l)}\right)$, input subsidy rate $\left(i^{(j)}\right)$, predator mortality rate $\left(\delta^{(j, l)}\right)$, and proportion of predators surviving migration $\left(\lambda^{(j, l)}\right)$ play in the PPS population dynamics.

In Section 3.2, we introduce a subsidy into the predator-prey model for $n$-Patch Model. For stable equilibria dynamics, introducing a subsidy into the system benefited the predator, but may or may not be detrimental to the prey (depending on the parameters taken) since there may be greater predation pressure. Therefore the extra nutrition in the system was sometimes mitigated by the damage caused to the prey. For a large enough subsidy input rate, stable equilibria will replace the limit cycles. The predator population has greater benefits from an increase of the subsidy input rate after the prey is extinct, because the predator has more food from the increased subsidy input rate in such a case.

In Section 3.3, we study the dynamics of the PPS model for all networks with a set of the carrying capacity values and subsidy input rates. This was previously studied in [23] for one and two-patch cases. There are similar $i-k$ bifurcation diagrams for all our network structures. The dynamics in the bifurcation diagram are those of predator-free equilibria, prey-free equilibria, positive equilibria, non-equilibrium dynamics (including, for example, limit cycles) involving predator, prey, and subsidy. We found the system will move from a positive equilibrium state to a non-equilibrium state as the carrying capacity increases. This process is known as the paradox of enrichment $[23,20,5,6,25,28]$.

With our observation, we are able to distinguish the large time dynamics as they depend on carrying capacity $(k)$ and subsidy input rate $(i)$. It is possible to obtain an equilibrium state of the system and avoid the extinction of the prey and predator if we can select suitable values of $i$ and $k$. Also, we realized 
that instability can be the result of nutritional enrichment under certain conditions specified in Section 3.3. In Luckinbill's experiment, the predator Didinium Nasutum consumed all the of the prey Paramecium Aurelia in a few hours before going extinct in a cerophyl nutrient medium [18]. Even with a thickened medium of methyl cellulose, the predator went through a few unstable oscillations before both species went extinct in two weeks [18]. Harrison built on this experiment and concluded that a thicker medium with cellulose less prey would shift the system to a stable state [7]. Veilleux's experiment with the Didinium and Paramecium also concluded that the concentration of the cerophyl nutrients also contributed to the stability of the ecosystem [32]. With low cerophyl, the predator and prey were able to coexist, with higher and higher population oscillations as the cerophyl level increased [32]. The last two experiments showed that either fewer prey or less nutrients in the ecosystem will result in a higher probability of the two species co-existing $[18,32]$. Therefore, there results suggested that attempting to enrich the ecosystem to increase food yield must be approached with caution. There is risk that this activity may result in decimation of the species [25]. Our numerical simulations are in agreement with these experimental findings.

We analyzed the predator migration rate effect between patches on the dynamics for different case conditions on different networks. We examine the effect of the predator migration rate with different carrying capacity values, predator mortality rates, proportion of predators surviving migration, and input rate of the subsidy in Sections 3.4, 3.5, 3.6, and 3.7, respectively. In Section 3.4, networks with various initial conditions show that as $\alpha$ increases, predators have a higher possibility to go extinct. This may be due to predators spending too much time moving through regions poor in food sources. When predators do not have sufficient food, $P_{7}$ is the most favourable for the predator. On the other hand, more predator migration stabilizes the system and prevents predators from going extinct when predators have a sufficient food source as allocated in other initial conditions. In such cases, the dynamics for the five networks considered are similar, but the dynamics for $C i_{10}(1,2)$ are slightly more favourable for coexistence.

For an ecosystem with a given carrying capacity, we are able to obtain the corresponding dynamics for different networks. We can then figure out which type of network is the most favourable for the coexistence of different species. Also, introducing new food sources to approachable patches can lower the extinction rate of the predators, which can increase the stability of the system. If a particular ecosystem is not stable from lack of food, we can use these studies to increase the food supply to where it is needed most.

In Section 3.5, we found that with a minimal carrying capacity, predators can only survive with very low death rates. There is no strong correlation between network structure and the results when $k$ is small. With a larger carrying capacity, the predators survive more easily, even with higher mortality rates. If the predator mortality rate is low, more predator movement will normally stabilize the system, assuming no deaths due to predators' migration. With a sufficient food source and larger carrying capacity, $T$ and $H$ are more suitable for coexistence compared with other networks.

In Section 3.6, when the carrying capacity is low and the food source is insufficient, the system only has positive equilibrium dynamics when the predator migration rate is low, regardless of the migration survival rate. If the food source is sufficient, we find for some networks that more movement will stabilise the system when the predator migration survival rate is higher than 0.95 . This means that in some cases, when predator death would occur in a single patch model, having multiple patches can actually allow the predator population to survive. This is an important finding, as it suggests that in some cases, added mobility will help (rather than hinder) predator populations, allowing them to survive or coexist when they would otherwise go extinct if confined to one location. We observed this behavior for the network choice $H$, although we hypothesise that this behavior would be possible for other kinds of networks, given that appropriate parameter values are selected.

In Section 3.7, we examined the effect of predators travelling between patches with different subsidy input rates and found some exciting results. For $K_{10}$, increasing $\alpha$ (for small enough i) acts to destabilize the dynamics of the system, causing the coexistence equilibrium to move into a limit cycle, similar to when we increase $k$ and find a similar bifurcation (paradox of enrichment). Therefore, the additional movement results in dynamics that mirror the paradox of enrichment results. Note that no results showing that increased movement causes limit cycles exist in the predator-prey literature. Therefore, increased movement resulting in observations similar to paradox of enrichment results is very exciting. Other networks do not show this behaviour, so it seems that a destabilization of coexistence equilibria to limit cycles through movement occurs for only specific families of networks. This result illustrates that the underlying network structure is vital in determining predator-prey dynamics. Note that for large enough subsidy input rates, stable preyfree equilibria replace limit cycles. This was observed even for the simpler island models considered in Nevai and Van Gorder [23].

One could extend this work in several ways. First, we can investigate the dynamics of predator-prey-subsidy system on other potential network structures that are of more real-world relevance. One might use these network structures for conservation effects to protect the environment. Second, our analysis is applicable to any other system that has predator-prey-subsidy ecosystems. Another example of a predator-prey-subsidy system is polar bear (predator), seal (prey) and carcasses of whales (subsidy) [35]. Also, we can consider a model of five coupled ODEs, describing the dynamics between polar bears (predator), seals (quarry), lemmings (prey), arctic foxes (scavenger), and seal carrions (subsidy). The polar bears and seals can be used to obtain a predator-prey relationship, and the other three as part of a similar predator-prey-subsidy equation. The input rate of the subsidy can be replaced by a lower prey consumption rate in certain models [15]. Finally, instead of using constant parameters, we can use time-dependent parameters to include seasonality in the model. For example, the subsidy input rate with the seal carrion can increase during the winter due to increased predation, while the subsidy decay rate increases during 
the summer due to higher seal carrion death rates.

\section{References}

[1] P. A. Abrams, "The evolution of predator-prey interaction: Theory and evidence," Annu. Rev. Ecol. Syst., vol. 31, no. 11, pp. 75-105, 2000.

[2] F. J. Ayala, M. E. Gilpin, and J. G. Ehrenfeld, "Competition between species: Theoretical models and experimental tests," Theor. Popul. Biol., vol. 14 , no. 3, pp. 331-356, 1973.

[3] A. A. Berryman, "The origins and evolution of predator-prey theory," Ecology, vol. 73, no. 5, pp. 1530-1535, 1992.

[4] G. F. Fussmann, S. P. Ellner, K. W. Shertzer and N. G. Hairston, "Crossing the Hopf bifurcation in a live predatorprey system," Science, vol. 290, no. 5495, pp. 1358-1360, 2000.

[5] M. Gilpin and M. Rosenzweig, "Enriched predatorprey systems: Theoretical stability," Science, vol. 177, no. 4052, pp. 902-904, 1972.

[6] W. S. C. Gurney and R. M. Nisbet, Ecological Dynamics, New York, Oxford: Oxford University Press, 1998.

[7] G. W. Harrison, "Comparing predatorprey models to Lukinbills experiment with Didinium and Paramecium," Ecology, vol. 76, no. 2, pp. 357374 , 1995.

[8] M. D. Holland and A. Hastings, "Strong effect of dispersal network structure on ecological dynamics," Nature, vol. 456, no. 7223, p. 792, 2008.

[9] C. S. Holling, "The components of predation as revealed by a study of small mammal predation of the European pine sawfly," Canadian Entomologist, vol. 91, no. 5, pp. 293-320, 1959.

[10] C. S. Holling, "The functional response of invertebrate predators to prey density," Memoirs Entomological Soc. Canada, no. 48, pp. 5-86, 1966.

[11] E. E. Holmes, M. A. Lewis, J. E. Banks, and R. R. Veit, "Partial differential equations in ecology: Spatial interactions and population dynamics," Ecology, vol. 75, no. 1, pp. 17-29, 1994.

[12] C. B. Huffaker, H. P. Shea and S. G. Herman, "Experimental studies on predation: complex dispersion and levels of food in an acarine predatorprey interaction," Hilgardia, vol. 34, pp. 305-330, 1963.

[13] P. Kareiva, A. Mullen, and R. Southwood, "Population dynamics in spatially complex environments: Theory and data [and discussion]," Philosophical Trans. Royal Soc. B: Biol. Sci., vol. 330, no. 1257, pp. 175-190, 1990.

[14] S. A. Levin, "Population dynamic models in heterogeneous environments," Annual Rev. Ecol. Syst., vol. 7, no. 11, pp. 287-310, 1976.

[15] D. Levy, H. A. Harrington, R. A. Van Gorder, "Role of seasonality on predator-prey-subsidy population dynamics," J. Theor. Biol., vol. 396, pp. 163-181, 2016.

[16] S. L. Lima, "Putting predators back into behavioral predator-prey interactions," Trends Ecol. Evol., vol. 17, no. 2, pp. 70-75, 2002.

[17] A. J. Lotka, "Elements of Physical Biology," Baltimore: Williams \& Wilkins, 1925.

[18] L. S. Luckinbill, "Coexistence in laboratory populations of Paramecium aurelia and its predator Didinium nasutum," Ecology, vol. 54, no. 6, pp. 1320-1327, 1973.

[19] MATLAB, Version 8.4 (R2014b), The MathWorks Inc., Natick, Massachusetts, 2014.

[20] R. M. May, "Limit cycles in predatorprey communities," Science, vol. 177, no. 4052, pp. 900-902, 1972.

[21] E. S. Minor and D. L. Urban, "Graph theory as a proxy for spatially explicit population models in conservation planning," Ecological Appl., vol. 17, no. 6, pp. 1771-1782, 2007.

[22] J. D. Murray, "Continuous Population Models for Single Species," Mathematical Biology 1: An Introduction, 3rd ed. New York: Springer, 2002, ch. 1 , sec. 1

[23] A. L. Nevai and R. A. Van Gorder, "Effect of resource subsidies on predator-prey population dynamics: a mathematical model," J. Biol. Dynamics, vol. 6, no. 2, pp. 891-922, 2012.

[24] P. Prestrud, "Adaptions by the arctic fox (Alopex logopus) to the polar winter," Arctic, vol. 44, no. 2, pp. 132-138, 1991.

[25] M. Rosenzweig, "The paradox of enrichment: Destabilization of exploitation ecosystems in ecological time," Science, vol. 171, no. 3969, pp. 385$387,1971$.

[26] J. D. Roth, "Temporal variability in arctic fox diet as reflected in stablecarbon isotopes; the importance of sea ice," Oecologia, vol. 133, no. 1, pp. 70-77, 2002.
[27] J. D. Roth, "Variability in marine resources affects arctic fox population dynamics," J. Anim. Ecol., vol. 72, no. 4, pp. 668-676, 2003.

[28] S. Roy and J. Chattopadhyay, "The stability of ecosystems: A brief overview of the paradox of enrichment," J. Biosci, vol. 32, no. 2, pp. 421428, 2007.

[29] M. Seymour and F. Altermatt, "Active colonization dynamics and diversity patterns are influenced by dendritic network connectivity and species interactions," Ecol. and Evol., vol. 4, no. 8, pp. 1243-1254, 2014.

[30] A. Sih, "Prey refuges and predator-prey stability," Theor. Popul. Biol., vol. 31, no. 1, pp. 1-12, 1984.

[31] M. E. Solomon, "The natural control of animal population," J. Anim. Ecol., vol. 18, no. 1, pp. 1-35, 1949.

[32] B. G. Veilleux, "An analysis of the predatory interaction between Paramecium and Didinium," J. Anim. Ecol., vol. 48, no. 3, pp. 787-803, 1979.

[33] M. Vogels, R. Zoeckler, D. Stasiw, and L. Cerny, “P. F. Verhulst's notice Sur La Loi Que La Populations Suit Dans Son Accroissement from Correspondence Mathematique Et Physique. Ghent, Vol. X, 1838," J. Biol. Phys., vol. 3, no. 4, pp. 183-192, 1975.

[34] V. Volterra, "Variation of fluctuations of the number of individuals in animal species living together," ICES J. Marine Sci., vol. 3, no. 1, pp. 3-51, 1928.

[35] World Wild Fund For Nature. "Polar bear diet," Internet: http://wwf.panda.org/what_we_do/where_we_work/arctic/wildlife/polar_bear/diet/, [Aug. 28, 2016].

[36] R. E. Wrigley and D. R. M. Hatch, "Arctic fox migrations in Manitoba," Arctic, vol. 29, no. 3, pp. 147-158, 1976. 